\title{
DETERMINACIÓN DE LA VIDA ÚTIL DE UN OLEODUCTO EN SERVICIO
}

\section{Determination of the lifespan of a Pipeline in Service}

\section{José Luis Soto Trinidad}

Resumen: Este trabajo describe la realización de un estudio de predicción de vida de un oleoducto en servicio basado en las teorías y métodos de la mecánica de la fractura probabilística. La tubería es de acero con especificación APIL 5L, tiene un diámetro exterior de $203.2 \mathrm{~mm}$ y una longitud de 72 kilómetros. Para llevar a cabo el estudio se inspeccionó y analizó la superficie y las uniones soldadas del ducto utilizando un equipo de ultrasonido. En consecuencia, en la superficie del oleoducto se detectaron defectos, tales como grietas, socavaduras, oxidación y corrosión. Además se midió el espesor de la tubería. Entonces, a partir de los datos obtenidos de la medición de los defectos, presión, propiedades mecánicas y espesor remanente del oleoducto se determinaron las dimensiones de las grietas y la vida útil del mismo.

Palabras claves: Vida útil, defectos, velocidad de propagación y mecánica de la fractura. 
Abstract: This paper deals with a study of life prediction of a pipeline in service based on the theories and methods of probabilistic fracture mechanics. The steel pipe is APIL 5L specification, has an outer diameter of $203.2 \mathrm{~mm}$ and a length of 72 kilometers. To conduct the study, the surface and pipeline welded joints were inspected and analyzed using ultrasound equipment. So, defects such as cracks, undercuts, oxidation and corrosion were detected on the surface of the pipeline. In addition, the thickness of the pipe was measured. Then, dimensions cracks and life were determined from the data obtained from the measurement data of the defects, pressure, mechanical properties and remaining thickness of pipe.

Keywords: Lifespan, defects, propagation velocity and fracture mechanics.

\section{Introducción}

En esta investigación se aborda un estudio de integridad estructural de una tubería de petróleo para determinar su vida útil y su confiabilidad estructural en servicio, por medio del uso de las teorías de la mecánica de la fractura probabilística. En este plantean las ecuaciones que conducen a obtener las variables de respuestas después del empleo de la técnica de ultrasonido para la identificación y evaluación de defectos a fin de tener los datos de entradas del estudio.

El oleoducto ha sido diseñado para transportar petróleo crudo desde una estación de bombeo hasta la Planta Principal, la cual está localizada a setenta y dos (72) kilómetros con respecto a la 
estación mencionada. La presión de diseño de la tubería es de $9.928 \mathrm{MPa}$ y cuenta con seis (6) válvulas de bloqueo distribuidas a lo largo para minimizar los daños en caso de derrame. Sus especificaciones son: $203.2 \mathrm{~mm}$ de diámetro exterior y el espesor de pared es de $5.563 \mathrm{~mm}$ para una especificación API 5 LX, Grado X-42, sin costura o soldadura por resistencia eléctrica.

En el estudio se tomó en cuenta la idea de que las grietas existentes en las partes de la estructura en servicio, pudieran crecer mientras esté en trabajo, lo que ha conducido a la frase descriptiva "diseño tolerante al daño". El enfoque de la filosofía se concentra en el crecimiento de grietas hasta que este se vuelve crítico y la parte se retira del servicio, en este caso, la herramienta de análisis es la mecánica de la fractura lineal elástica (MFEL), la inspección y el mantenimiento son esenciales en la decisión de retirar partes antes de que la grieta alcance un tamaño catastrófico, tal como se ha hecho en los trabajos de reparación del ducto (Irwin, 1957) (Budynas y Nisbett, 2008). Por lo tanto, como la seguridad humana está involucrada, se han hecho inspecciones periódicas en busca de grietas (Askeland, D. \& Phulé).

En el estudio, el uso de factores de concentración del esfuerzo proporciona una indicación de la carga promedio que se requiere sobre una parte para que ocurra la deformación plástica, o la fluencia; estos factores también son útiles para analizar las cargas sobre una parte que podrían causar fractura por fatiga (Shigley \& Mischke, 1990). Al combinar el análisis de los cambios elásticos grandes en una estructura o parte que ocurren a medida que una grieta delgada crece, con mediciones de la energía que se requiere para producir nuevas superficies de fractura, es posible calcular el esfuerzo promedio (si no existieran grietas) que causaría el crecimiento de una grieta en una parte (Ferrer \& Amigo, 2005). Tal cálculo, solamente, se permite en partes con grietas para las cuales se ha completado el análisis elástico y para materiales frágil (Smith \& Javad, 2005). En el caso de la tubería que se analiza, es cilíndrica de pared delgada, por lo que constituye una aplicación 
importante en el análisis de esfuerzo plano. Sus paredes oponen poca resistencia a la flexión, si puede suponerse que las fuerzas internas ejercidas sobre una parte de la pared son tangentes a la superficie del tubo, entonces, puede decirse que los esfuerzos resultantes están contenidos en un plano tangente a la superficie de dicho ducto (Bell \& Johnson, 2007). También, se utilizará la ecuación de París para estudiar la predicción de vida dicho oleoducto porque correlaciona el crecimiento linealizado de cada una de las grietas que se originen en el mismo (Ferrer \& Amigo, 2005), (González, 2004). A continuación se describe el procedimiento metodológico utilizado en el estudio y, posteriormente, los resultados obtenidos.

\section{Procedimiento experimental}

En este estudio de integridad basado en la mecánica de la fractura, se ha hecho un análisis de esfuerzos fundamentado en recipientes de pared delgada, el cual se limita al de recipientes cilíndricos (Martínez, et al., 2000), (Erdogan, 2000). Por lo tanto, se consideró el oleoducto como un recipiente cilíndrico de radio interior $r$ y espesor de pared $e$, que contiene el fluido (petróleo) a presión. Considerando que se ejercen esfuerzos sobre un pequeño elemento de pared con lados respectivamente paralelos y perpendiculares al eje de la tubería. Estos esfuerzos normales $\sigma_{1}$ y $\sigma_{2}$ son los esfuerzos principales (Irwin, 1957). El esfuerzo $\sigma_{1}$ se conoce como esfuerzo de costilla y el esfuerzo $\sigma_{2}$ es el esfuerzo longitudinal (Bell \& Johnson, 2007). Para determinar los esfuerzos de costilla $\sigma_{1}$ se hizo la suposición de separación de una porción del recipiente y su contenido se limitó por el plano $x y$ y por dos planos paralelos al plano $y z$ con una distancia $\Delta x$ de separación entre ellos. Las fuerzas paralelas al eje $Z$ que estaban actuando en el cuerpo libre así definido, consisten en las fuerzas internas elementales $\sigma_{1} d A$ en las secciones de pared y en las fuerzas de presión elementales $p d A$ ejercidas sobre la porción de fluido. Donde $p$ es la presión manométrica del fluido, que representa 
el exceso de la presión interior sobre la presión atmosférica exterior. Entonces, la resultante de las fuerzas internas $\sigma_{1} d A$ es igual al producto de $\sigma_{1} \mathrm{y}$ del área transversal $2 e \Delta x$ de la pared, mientras que la resultante de las fuerzas $p d A$ es el producto de $p$ y el área $2 r \Delta x$. Escribiendo la ecuación de equilibrio, se tiene:

$$
\sum F_{z}=0: \sigma_{1}(2 e \Delta x)-p(2 r \Delta x)=0 \rightarrow e c .(1)
$$

Resolviendo para el esfuerzo de costilla $\sigma_{1}$

$$
\sigma_{1}=\frac{p r}{e} \rightarrow e c .(2)
$$

Para determinar el esfuerzo longitudinal $(\sigma 2)$ se hizo un corte perpendicular al eje x y se consideró el cuerpo libre que consta de la parte del recipiente y de su contenido a la izquierda de la sección. Las cargas que están actuando en este cuerpo libre son las fuerzas internas $(\sigma 2 d A)$ en la sección de pared y la presión $(p d A)$ ejercida sobre la porción de fluido. El área de la sección de fluido es $\pi r_{2}$, la circunferencia del cilindro $(2 \pi r)$ por su espesor de pared $(e)$ se han agrupado en una ecuación de equilibrio (ec. 3) (Bell \& Johnson, 2007):

$$
\sum F_{x}=0: \sigma_{2}(2 \pi r e)-p\left(\pi r^{2}\right)=0 \rightarrow e c .(3)
$$

Y despejando para el esfuerzo longitudinal $\sigma_{2}$ :

$$
\sigma_{2}=\frac{p r}{2 e} \quad \rightarrow e c .(4)
$$

Asimismo, se sabe que este estudio implica la evaluación del número de ciclos del crecimiento de grietas hasta la fractura $N_{c}$. Por consiguiente, el número de ciclos total del servicio de la tubería hasta la rotura $\left(N_{t}\right)$, abarca los ciclos de la iniciación de la grieta $\left(N_{g}\right)$, y los ciclos de su crecimiento $\left(N_{c}\right)$, de donde se tiene el siguiente modelo matemático (Ferrer \& Amigo, 2005): 


$$
N_{t}=N_{g}+N_{c} \rightarrow \text { ec. (5) }
$$

El análisis por separado requerido para la iniciación y el crecimiento de una grieta se debe al diferente nivel de tensión en la superficie de entalla, antes y después de iniciarse una fisura $\sigma_{f}$ y $\sigma_{f g}$. Entonces, el modelo aplicable para evaluar la tenacidad a la fractura desde antes de iniciarse cualquier grieta se describe con la ecuación 6 (Ferrer \& Amigo, 2005):

$$
\Delta K=F_{f} \cdot \Delta \sigma \cdot \sqrt{\pi \cdot a} \quad \rightarrow e c \cdot(6)
$$

Esta ecuación involucra la tensión a que se somete el ducto $(\sigma)$ en cada parte, la longitud de la grieta $(a)$ y un factor geométrico que depende de la ubicación del defecto $\left(F_{f}\right)$.

En el caso de para la predicción de vida del oleoducto se ha establecido la correlación analítica de cada una de las grietas que se manifiesten en este, por lo tanto, para obtener la solución de esta problemática se aplicó el modelo matemático de París debido a que correlaciona el crecimiento linealizado de una grieta en la etapa II con la pendiente $n$. Esta etapa II, ubicada entre la etapa I (crecimiento estable rápido) y la etapa III (crecimiento inestable y rápido), es justificada por la teoría de fatiga (Ferrer \& Amigo, 2005), (González, 2004).

$$
d a / d N=C(\Delta K)^{n} \rightarrow \text { ec. }(7)
$$

Donde los factores $C$ y $n$, son parámetros característicos del material y del entorno considerado, y dependen de la relación $R_{f}=\sigma_{\min } / \sigma_{\max }$. Luego, se integra la ecuación de París para calcular el número de ciclos entre dos estados en el proceso de crecimiento de la grieta de fatiga; de esta manera, queda implícito el concepto de límite de fatiga que corresponde a los valores de $\Delta K<\Delta K_{I S}$ donde inicia la grieta $\left(\Delta K_{I S}\right)$, por debajo del cual no existe formación de grieta de fatiga y se determina el crecimiento inestable de una grieta $\left(\Delta_{I C}\right)$ (Ferrer \& Amigo, 2005). 
Por último, se evaluaron los resultados experimentales obtenidos por el análisis de ultrasonido (Ferrer \& Amigo, 2005) y determinísticos a través de métodos y herramientas estadísticas y de probabilidad para encontrar las funciones de distribución a que mejor se ajustan (Walpole \& Myers, 1992), (Mendenhall \& Sincich, 1997) y mediante el programa@Risk Palisade Corporation (2002) hallar la función de supervivencia o la probabilidad de que la tubería estudiada no se fracture bajo las condiciones de servicio en que ha estado operando.

\section{Resultados y discusiones}

Los resultados del estudio de la mecánica de la fractura probabilística (MFP) realizado al Oleoducto, se presentan a continuación.

\section{RESULTADOS DE LAS PRUEBAS POR ULTRASONIDOS EN EL DUCTO}

En la figura N. ${ }^{\circ} 1$, se muestra la realizacion de la medición de defectos y el espesor del Oleoducto.
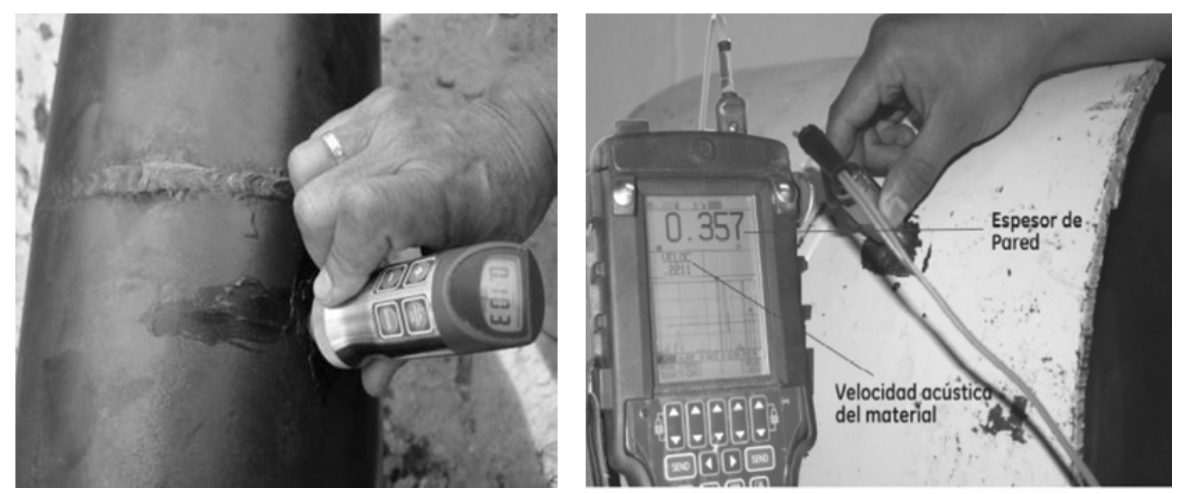

Figura N. ${ }^{\circ} 1$ - Medición de defectos y espesor del oleoducto de $\varnothing 8$ con un estudio por ultrasonido.

Los datos de las lecturas de los defectos, grosor y características en diferentes partes de la tubería (figura N. ${ }^{\circ} 1$ ), se presentan en la tabla N. ${ }^{\circ}$ 1. La presión de diseño (Pd) de esta es de 9.928 MPa. 
Tabla N. ${ }^{\circ} 1$

Resultados obtenidos por análisis de ultrasonido en el Oleoducto

\begin{tabular}{|c|c|c|c|c|c|}
\hline $\begin{array}{c}\text { Longitud tubo } \\
\text { con defecto } \\
(\mathbf{m})\end{array}$ & $\begin{array}{c}\text { Profundidad } \\
\text { del defecto } \\
(\mathbf{m m})\end{array}$ & $\begin{array}{c}\text { Espesor re- } \\
\text { manente de } \\
\text { pared (mm) }\end{array}$ & $\begin{array}{c}\text { Longitud } \\
\text { del defecto } \\
(\mathbf{m m})\end{array}$ & $\begin{array}{c}\text { Ancho del } \\
\text { defecto } \\
(\mathbf{m m})\end{array}$ & $\begin{array}{c}\text { Espesor de } \\
\text { Pared } \\
(\mathbf{m m})\end{array}$ \\
\hline 9.187 & 1.58 & 4.14 & 268 & 140 & 5.72 \\
\hline 12.273 & 1.38 & 4.34 & 482 & 183 & 5.72 \\
\hline 12.599 & 1.38 & 4.34 & 185 & 65 & 5.72 \\
\hline 12.670 & 1.38 & 3.95 & 205 & 140 & 5.33 \\
\hline 12.223 & 1.38 & 3.95 & 325 & 54 & 5.33 \\
\hline 12.692 & 2.37 & 3.16 & 398 & 129 & 5.53 \\
\hline 12.629 & 1.58 & 3.95 & 165 & 118 & 5.53 \\
\hline 12.803 & 1.97 & 3.36 & 465 & 226 & 5.33 \\
\hline 12.730 & 1.58 & 3.95 & 350 & 108 & 5.53 \\
\hline 12.652 & 1.78 & 3.55 & 845 & 140 & 5.33 \\
\hline 11.836 & 1.39 & 4.14 & 243 & 151 & 5.53 \\
\hline 12.317 & 1.58 & 3.75 & 200 & 151 & 5.33 \\
\hline 12.457 & 1.39 & 4.14 & 915 & 161 & 5.53 \\
\hline 12.423 & 1.39 & 4.14 & 385 & 97 & 5.53 \\
\hline 12.599 & 1.58 & 3.75 & 213 & 129 & 5.33 \\
\hline 12.341 & 1.58 & 3.75 & 513 & 129 & 5.33 \\
\hline 12.508 & 1.58 & 3.75 & 232 & 97 & 5.33 \\
\hline 12.891 & 2.17 & 3.16 & 242 & 172 & 5.33 \\
\hline 5.702 & 1.58 & 3.95 & 292 & 65 & 5.53 \\
\hline 12.703 & 1.58 & 3.95 & 338 & 172 & 5.53 \\
\hline 13.414 & 1.78 & 3.55 & 762 & 204 & 5.33 \\
\hline 1.528 & 1.39 & 4.14 & 172 & 129 & 5.53 \\
\hline 12.925 & 1.39 & 4.14 & 397 & 204 & 5.53 \\
\hline 12.685 & 1.39 & 4.14 & 885 & 129 & 5.53 \\
\hline 12.655 & 1.38 & 3.95 & 305 & 323 & 5.33 \\
\hline 12.667 & 1.58 & 3.95 & 238 & 194 & 5.53 \\
\hline 13.235 & 1.78 & 3.55 & 190 & 75 & 5.33 \\
\hline 13.159 & 1.58 & 3.75 & 725 & 194 & 5.33 \\
\hline 12.990 & 1.38 & 3.95 & 357 & 97 & 5.33 \\
\hline 12.745 & 1.39 & 4.14 & 250 & 129 & 5.53 \\
\hline 12.295 & 1.38 & 3.95 & 772 & 226 & 5.33 \\
\hline 12.548 & 1.58 & 3.95 & 970 & 215 & 5.53 \\
\hline 7.737 & 1.78 & 3.75 & 2205 & 312 & 5.53 \\
\hline 12.528 & 1.38 & 3.95 & 197 & 215 & 5.33 \\
\hline 12.971 & 1.58 & 3.75 & 478 & 258 & 5.33 \\
\hline 12.271 & 1.38 & 3.95 & 273 & 183 & 5.33 \\
\hline 12.409 & 1.38 & 3.95 & 752 & 280 & 5.33 \\
\hline & & & & & \\
\hline
\end{tabular}


Los esfuerzos ejercidos sobre el ducto debido a la simetría axial de dicho recipiente y de su contenido muestran que no se ejercen esfuerzos cortantes sobre el elemento de acuerdo a teoría del esfuerzo plano (Irwin, 1957) (Bell \& Johnson, 2007). Los esfuerzos normales $\sigma_{1}$ y $\sigma_{2}$ presentados en la tabla $\mathrm{N} .^{\circ} 2$, son los esfuerzos principales que se determinaron. El esfuerzo $\sigma_{1}$ es la tensión máxima de trabajo, que se conoce como esfuerzo de costilla, y el esfuerzo $\sigma_{2}$ es el esfuerzo longitudinal, ambos esfuerzos influyen en el comportamiento mecánico del oleoducto y se describen por la distribución de probabilidad logística obtenida mediante el programa@Risk Palisade Corporation (2002) (figura N. . 2).
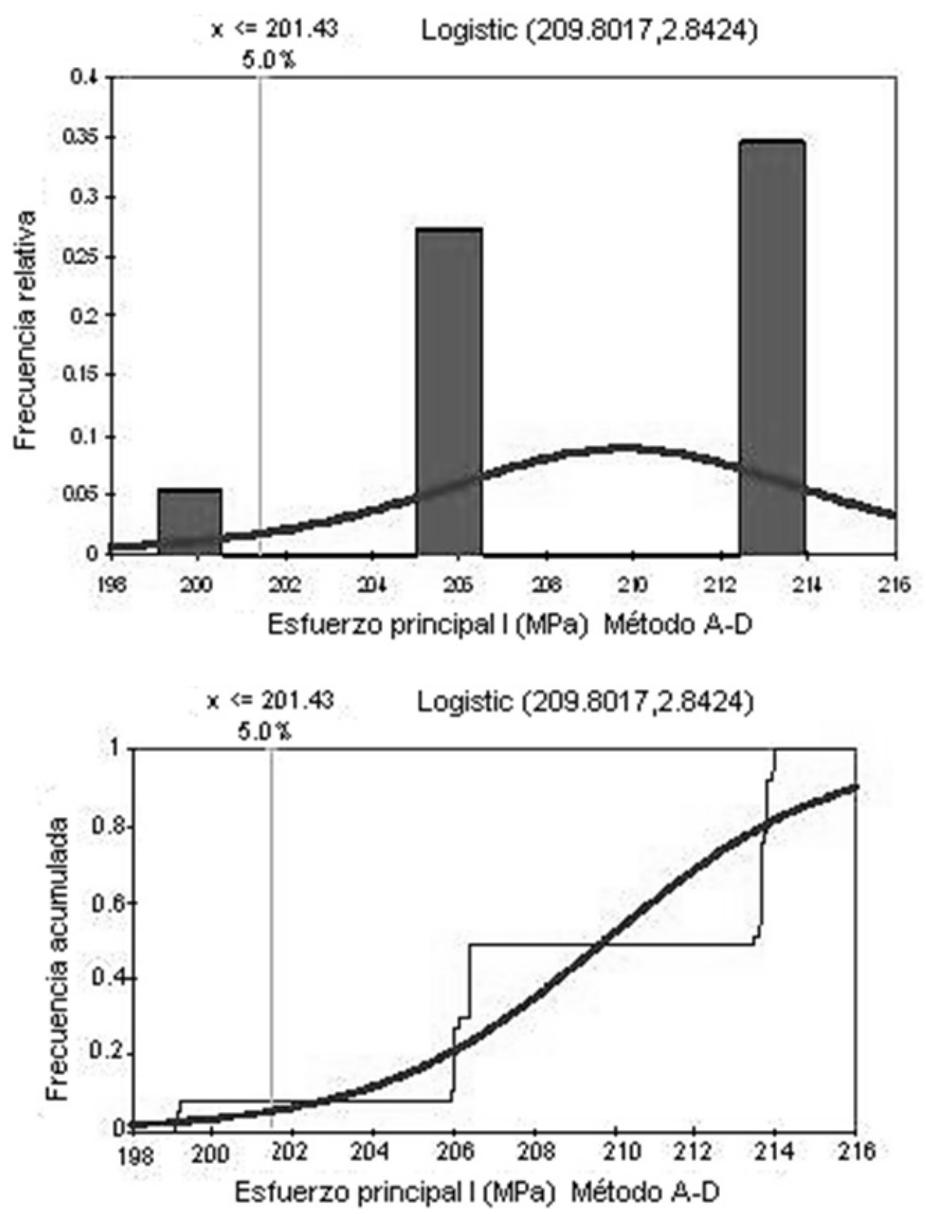

Ciencia y Sociedad 2013; 38(2): 405-428 


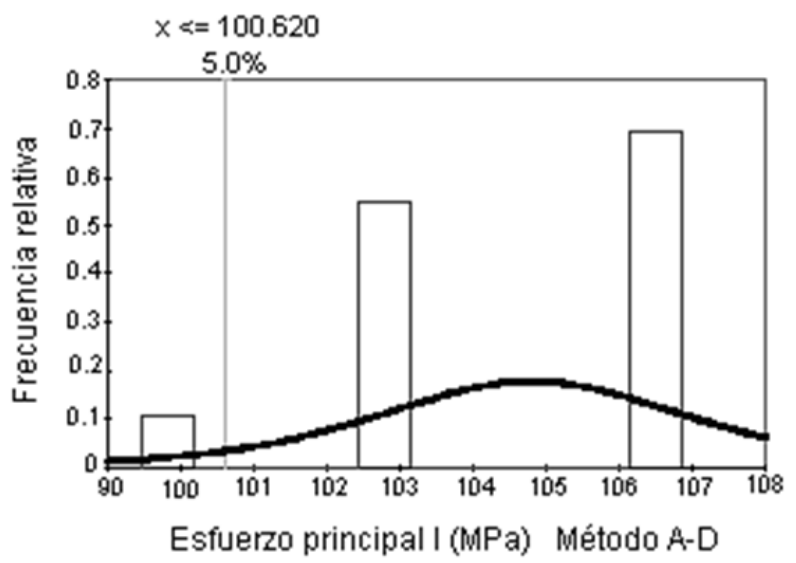

Logistic (104.8003, 1.4198)

$x<=100.620$

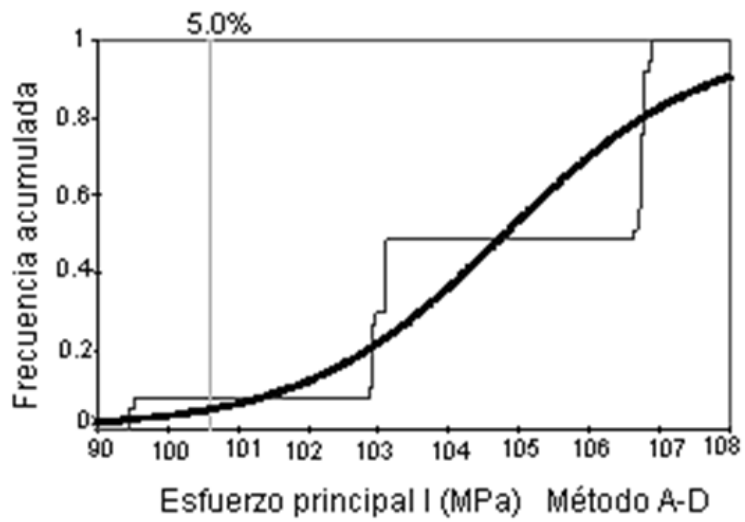

Figura N. ${ }^{\circ} 2$

Distribución logística de los esfuerzos que actúan en el Oleoducto

Las fuerzas que actúan en el oleoducto son las fuerzas internas $\sigma_{2} d A$ en la sección de pared y las fuerzas de presión $p d A$ ejercidas sobre el fluido (Bell \& Johnson, 2007). El área de la sección de fluido es $\pi r^{2}$ y circunferencia $2 \pi r$ del cilindro por su espesor de pared $e$, da el equilibrio planteado por la ecuación 8. Entonces, el esfuerzo cortante máximo en la pared que se ajusta al comportamiento de una distribución logística (figura N. ${ }^{\circ}$ ), es igual al radio 
del círculo del diámetro y corresponde a una rotación de $45^{\circ}$ alrededor de un eje longitudinal y fuerza del plano de esfuerzo, es decir, dicha tensión cortante es igual al esfuerzo longitudinal.

Utilizando los datos de la tabla N. ${ }^{\circ} 1$ y sustituyéndolos en las ecuaciones 2 y 4 respectivamente, se calcularon los esfuerzos principales que inciden en el ducto con respecto a las presiones prensentadas en cada zona; los resultados se muestran en la tabla N. ${ }^{\circ}$ 2. El espesor remanente de la tubería es el mismo que se utilizó en los cálculos de estas tensiones.

\section{Tabla N. ${ }^{\circ} 2$}

Valores de los esfuerzos que se presentan en las zonas con defectos del Oleoducto

\begin{tabular}{|c|c|c|c|c|c|}
\hline $\begin{array}{c}\text { Long. Tubo } \\
\text { con defecto } \\
(\mathbf{m})\end{array}$ & $\begin{array}{c}\text { Presión } \\
\mathbf{( M P a )}\end{array}$ & $\begin{array}{c}\text { Esfuerzo } \\
\text { principal I } \\
\mathbf{( M P a )}\end{array}$ & $\begin{array}{c}\text { Esfuerzo } \\
\text { principal II } \\
\mathbf{( M P a )}\end{array}$ & $\begin{array}{c}\text { Esfuerzo } \\
\text { medio (MPa) }\end{array}$ & $\begin{array}{c}\text { Tensión } \\
\text { diseño (MPa) }\end{array}$ \\
\hline 9.187 & 7.902 & 199.227 & 99.518 & 149.373 & 250.325 \\
\hline 12.273 & 8.277 & 199.081 & 99.445 & 149.263 & 238.790 \\
\hline 12.599 & 8.277 & 199.081 & 99.445 & 149.263 & 238.790 \\
\hline 12.670 & 8.084 & 213.628 & 106.712 & 160.170 & 262.366 \\
\hline 12.223 & 8.084 & 213.628 & 106.712 & 160.170 & 262.366 \\
\hline 12.692 & 6.235 & 205.972 & 102.887 & 154.430 & 327.958 \\
\hline 12.629 & 7.796 & 206.011 & 102.907 & 154.459 & 262.366 \\
\hline 12.803 & 6.872 & 213.473 & 106.634 & 160.053 & 308.437 \\
\hline 12.730 & 7.796 & 206.011 & 102.907 & 154.459 & 262.366 \\
\hline 12.652 & 7.275 & 213.911 & 106.853 & 160.382 & 291.929 \\
\hline 11.836 & 8.185 & 206.367 & 103.085 & 154.726 & 250.325 \\
\hline 12.317 & 7.679 & 213.734 & 106.765 & 160.249 & 276.359 \\
\hline 12.457 & 8.185 & 206.367 & 103.085 & 154.726 & 250.325 \\
\hline 12.423 & 8.185 & 206.367 & 103.085 & 154.726 & 250.325 \\
\hline 12.599 & 7.679 & 213.734 & 106.765 & 160.249 & 276.359 \\
\hline 12.341 & 7.679 & 213.734 & 106.765 & 160.249 & 276.359 \\
\hline 12.508 & 7.679 & 213.734 & 106.765 & 160.249 & 276.359 \\
\hline 12.891 & 6.466 & 213.588 & 106.692 & 160.140 & 327.958 \\
\hline 5.702 & 7.796 & 206.011 & 102.907 & 154.459 & 262.366 \\
\hline
\end{tabular}




\begin{tabular}{|c|c|c|c|c|c|}
\hline $\begin{array}{c}\text { Long. Tubo } \\
\text { con defecto } \\
(\mathbf{m})\end{array}$ & $\begin{array}{c}\text { Presión } \\
\mathbf{( M P a )}\end{array}$ & $\begin{array}{c}\text { Esfuerzo } \\
\text { principal I } \\
\mathbf{( M P a})\end{array}$ & $\begin{array}{c}\text { Esfuerzo } \\
\text { principal II } \\
\mathbf{( M P a )}\end{array}$ & $\begin{array}{c}\text { Esfuerzo } \\
\text { medio (MPa) }\end{array}$ & $\begin{array}{c}\text { Tensión } \\
\text { diseño (MPa) }\end{array}$ \\
\hline 12.703 & 7.796 & 206.011 & 102.907 & 154.459 & 262.366 \\
\hline 13.414 & 7.275 & 213.911 & 106.853 & 160.382 & 291.929 \\
\hline 1.528 & 8.185 & 206.367 & 103.085 & 154.726 & 250.325 \\
\hline 12.925 & 8.185 & 206.367 & 103.085 & 154.726 & 250.325 \\
\hline 12.685 & 8.185 & 206.367 & 103.085 & 154.726 & 250.325 \\
\hline 12.655 & 8.084 & 213.628 & 106.712 & 160.170 & 262.366 \\
\hline 12.667 & 7.796 & 206.011 & 102.907 & 154.459 & 262.366 \\
\hline 13.235 & 7.275 & 213.911 & 106.853 & 160.382 & 291.929 \\
\hline 13.159 & 7.679 & 213.734 & 106.765 & 160.249 & 276.359 \\
\hline 12.990 & 8.084 & 213.628 & 106.712 & 160.170 & 262.366 \\
\hline 12.745 & 8.185 & 206.367 & 103.085 & 154.726 & 250.325 \\
\hline 12.295 & 8.084 & 213.628 & 106.712 & 160.170 & 262.366 \\
\hline 12.548 & 7.796 & 206.011 & 102.907 & 154.459 & 262.366 \\
\hline 7.737 & 7.405 & 206.113 & 102.958 & 154.535 & 276.359 \\
\hline 12.528 & 8.084 & 213.628 & 106.712 & 160.170 & 262.366 \\
\hline 12.971 & 7.679 & 213.734 & 106.765 & 160.249 & 276.359 \\
\hline 12.271 & 8.084 & 213.628 & 106.712 & 160.170 & 262.366 \\
\hline 12.409 & 8.084 & 213.628 & 106.712 & 160.170 & 262.366 \\
\hline
\end{tabular}

A partir de los datos de las tablas 1 y 2, empleando la ecuación seis, se calcularon los tamaños de grietas y con la ecuación siete se determinó la velocidad de propagación de las mismas, la cual dio lugar al cálculo de la vida del ducto en ciclos a partir de una grieta empleando la ecuación 5. La avería se detectó por el analisis de utrasonido; cuya dimensión es de $a_{i}=0.019 m$. Los valores de los tamaños y velocidad de propagación de las grietas así como su duración se presentan en la tabla N. ${ }^{\circ}$. 
Tabla N. 3 - Valores de vida, tamaño y velocidad de propagación de grietas en el Oleoducto

\begin{tabular}{|c|c|c|c|c|c|c|}
\hline $\begin{array}{l}\text { Longitud } \\
\text { Tubo con } \\
\text { daño (m) }\end{array}$ & $\begin{array}{c}\text { Factor de } \\
\text { intensidad } \\
\text { esfuerzo } \\
\Delta \mathrm{KI}(\mathrm{MPa})\end{array}$ & $\begin{array}{c}\mathrm{da} / \mathrm{dN} \\
\mathrm{mm} / \text { ciclos }\end{array}$ & $\begin{array}{c}\text { Grieta } \\
\text { máxima } \\
a_{c}(\mathrm{~m})\end{array}$ & $\begin{array}{c}\text { Grieta } \\
\text { mínima a } \\
\text { detectar } \\
a_{\min }(\mathrm{m})\end{array}$ & $\begin{array}{l}\text { Último } \\
\text { ciclo de } \\
\text { vida } \\
\text { (ciclos) }\end{array}$ & $\begin{array}{l}\text { Vida útil } \\
\text { (ciclos) }\end{array}$ \\
\hline 9.187 & 54.587 & 0.000008 & 0.023 & 0.015 & 3062.746 & 7921.601 \\
\hline 12.273 & 54.547 & 0.000008 & 0.023 & 0.016 & 3092.288 & 6169.604 \\
\hline 12.599 & 54.547 & 0.000008 & 0.023 & 0.016 & 3092.288 & 6169.604 \\
\hline 12.670 & 58.533 & 0.000010 & 0.020 & 0.013 & 673.403 & 6128.409 \\
\hline 12.223 & 58.533 & 0.000010 & 0.020 & 0.013 & 673.403 & 6128.409 \\
\hline 12.692 & 56.435 & 0.000009 & 0.022 & 0.008 & 1825.399 & 17113.366 \\
\hline 12.629 & 56.446 & 0.000009 & 0.022 & 0.013 & 1818.865 & 7901.596 \\
\hline 12.803 & 58.490 & 0.000010 & 0.020 & 0.010 & 694.492 & 11966.974 \\
\hline 12.730 & 56.446 & 0.000009 & 0.022 & 0.013 & 1818.865 & 7901.596 \\
\hline 12.652 & 58.610 & 0.000010 & 0.020 & 0.011 & 635.323 & 9771.166 \\
\hline 11.836 & 56.543 & 0.000009 & 0.021 & 0.015 & 1759.855 & 6131.650 \\
\hline 12.317 & 58.561 & 0.000010 & 0.020 & 0.012 & 659.182 & 7862.983 \\
\hline 12.457 & 56.543 & 0.000009 & 0.021 & 0.015 & 1759.855 & 6131.650 \\
\hline 12.423 & 56.543 & 0.000009 & 0.021 & 0.015 & 1759.855 & 6131.650 \\
\hline 12.599 & 58.561 & 0.000010 & 0.020 & 0.012 & 659.182 & 7862.983 \\
\hline 12.341 & 58.561 & 0.000010 & 0.020 & 0.012 & 659.182 & 7862.983 \\
\hline 12.508 & 58.561 & 0.000010 & 0.020 & 0.012 & 659.182 & 7862.983 \\
\hline 12.891 & 58.522 & 0.000010 & 0.020 & 0.008 & 678.881 & 14389.136 \\
\hline 5.702 & 56.446 & 0.000009 & 0.022 & 0.013 & 1818.865 & 7901.596 \\
\hline 12.703 & 56.446 & 0.000009 & 0.022 & 0.013 & 1818.865 & 7901.596 \\
\hline 13.414 & 58.610 & 0.000010 & 0.020 & 0.011 & 635.323 & 9771.166 \\
\hline 1.528 & 56.543 & 0.000009 & 0.021 & 0.015 & 1759.855 & 6131.650 \\
\hline 12.925 & 56.543 & 0.000009 & 0.021 & 0.015 & 1759.855 & 6131.650 \\
\hline 12.685 & 56.543 & 0.000009 & 0.021 & 0.015 & 1759.855 & 6131.650 \\
\hline 12.655 & 58.533 & 0.000010 & 0.020 & 0.013 & 673.403 & 6128.409 \\
\hline 12.667 & 56.446 & 0.000009 & 0.022 & 0.013 & 1818.865 & 7901.596 \\
\hline 13.235 & 58.610 & 0.000010 & 0.020 & 0.011 & 635.323 & 9771.166 \\
\hline 13.159 & 58.561 & 0.000010 & 0.020 & 0.012 & 659.182 & 7862.983 \\
\hline 12.990 & 58.533 & 0.000010 & 0.020 & 0.013 & 673.403 & 6128.409 \\
\hline 12.745 & 56.543 & 0.000009 & 0.021 & 0.015 & 1759.855 & 6131.650 \\
\hline 12.295 & 58.533 & 0.000010 & 0.020 & 0.013 & 673.403 & 6128.409 \\
\hline 12.548 & 56.446 & 0.000009 & 0.022 & 0.013 & 1818.865 & 7901.596 \\
\hline 7.737 & 56.473 & 0.000009 & 0.022 & 0.012 & 1801.961 & 9834.732 \\
\hline 12.528 & 58.533 & 0.000010 & 0.020 & 0.013 & 673.403 & 6128.409 \\
\hline 12.971 & 58.561 & 0.000010 & 0.020 & 0.012 & 659.182 & 7862.983 \\
\hline 12.271 & 58.533 & 0.000010 & 0.020 & 0.013 & 673.403 & 6128.409 \\
\hline 12.409 & 58.533 & 0.000010 & 0.020 & 0.013 & 673.403 & 6128.409 \\
\hline
\end{tabular}


En la predicción de vida remanente del ducto, una de las aplicaciones de mayor impacto de la mecánica de fractura es la predicción de la vida útil, que explica que cuando una grieta en dicho oleoducto se propaga, la resistencia disminuye hasta que dicha tubería no pueda resistir la carga de trabajo y falle (Ferrer \& Amigo, 2005), (González, 2004), esto se muestra en la figura N. ${ }^{\circ} 3$. Por lo tanto, la posibilidad que brinda la mecánica de fractura de predecir la rapidez de propagación de una grieta, es precisamente lo que hace que la predicción de vida sea posible (Shigley \& Mischke, 1990). Por ende, para predecir la vida de ducto, se determinó que una de las grietas detectadas ha de crecer (ecuación 8), desde su tamaño detectado hasta su tamaño crítico, es decir de su vida remanente, además se estudió su rapidez de propagación durante el servicio (Erdogan, 2000).
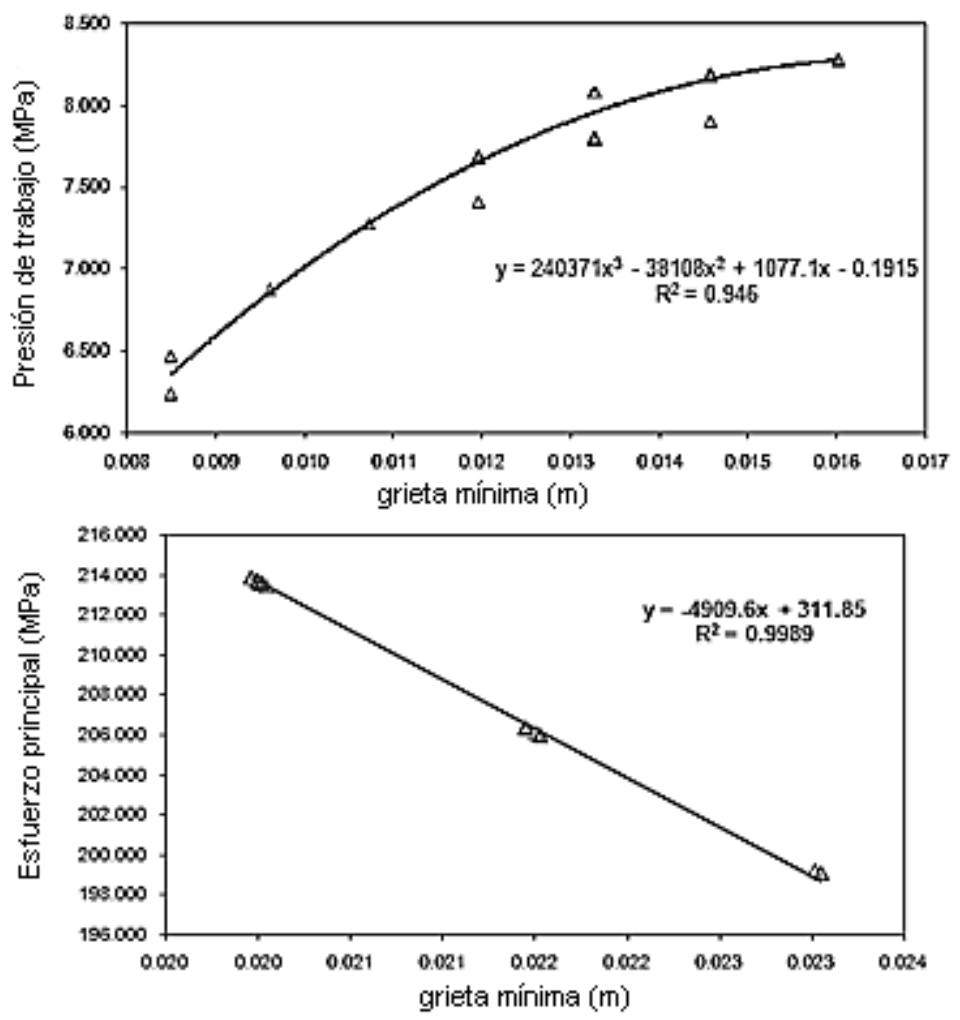

Figura N. 3 - Grietas mínima y crítica que se pueden originar bajo la presión de trabajo en el Oleoducto 
Un análisis de la figura N. ${ }^{\circ} 3$ permite conocer otros aspectos relevantes de la predicción de vida de la tubería, el ducto fue diseñado bajo la suposición de que el material no contenía defectos y la resistencia de diseño está determinada por las propiedades mecánicas de los materiales de fabricación y las características geométricas de dicho componente (Budinski, 1992), (Mangonon, 2001). De acuerdo a Ferrer \& Amigo (2005) cuando aparece una grieta, inicialmente esta no tiene un efecto en la resistencia residual, pero a medida que la grieta crece, la resistencia va disminuyendo (Fig. 3b). El tamaño de grieta que disminuye la resistencia por debajo del nivel de diseño es el tamaño mínimo detectado mediante el análisis (González, 2004) (Fig. 4).

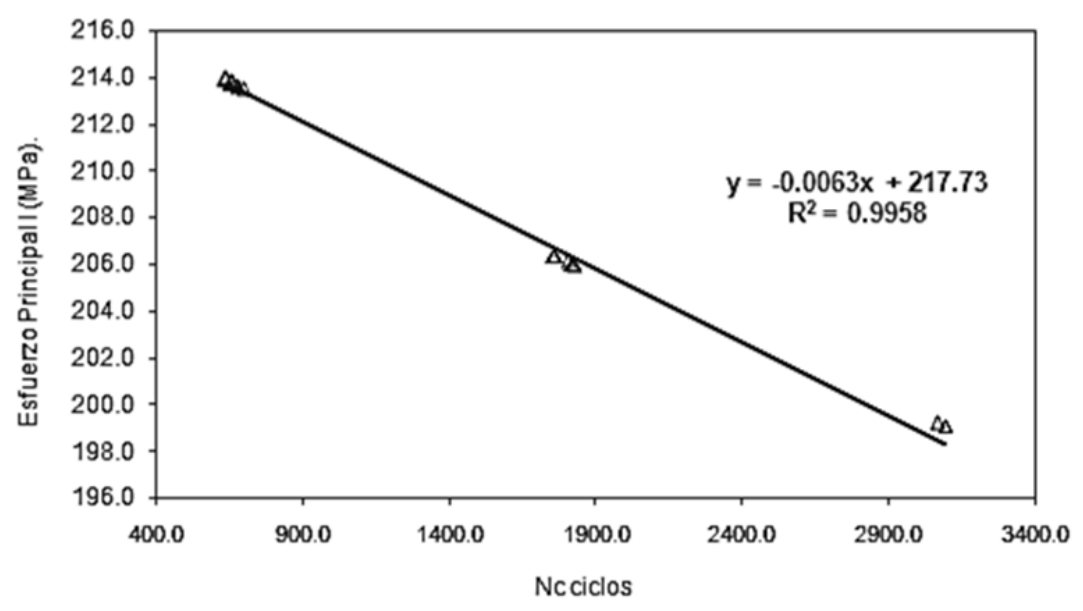

Figura N. ${ }^{\circ} 4$ - Tensión de trabajo $V s$ la vida del Oleoducto

A partir del tamaño mínimo a detectar y del tamaño crítico se obtuvo la vida útil del tubo, o sea, su vida máxima de operación. La vida remanente se interpreta como el tiempo que ha tardado en crecer la grieta desde el tamaño detectado en la inspección hasta el tamaño crítico (Anderson, 1995) (Fig. 3b). Sin embargo, puede ser que la posibilidad de fin de vida de operación, sea cuando la resistencia del material disminuya por efecto del servicio hasta quedar debajo de los niveles normales de operación, como muestra la figura N. ${ }^{\circ} 4$ (González, 2004). En este caso, la vida útil es determinada por el 
tiempo en que la resistencia del tubo disminuye desde su valor inicial hasta igualar el nivel normal de operación, cuyos valores se muestran en la tabla N. ${ }^{\circ}$ 3. La predicción de la vida útil en este caso, es más complicada por la dificultad de predicción de la rapidez de degradación de las propiedades del material -ya que las especificaciones de fabricación indican las propiedades mínimas requeridas y los materiales siempre poseen una resistencia mayor que el mínimo especificado (González, 2008). Las grietas desde su tamaño mínimo predictivo hasta su mayor tamaño presentan un comportamiento mecánico regido por una curva de distribución logística tal como se muestra en la figura N. ${ }^{\circ} 5$.

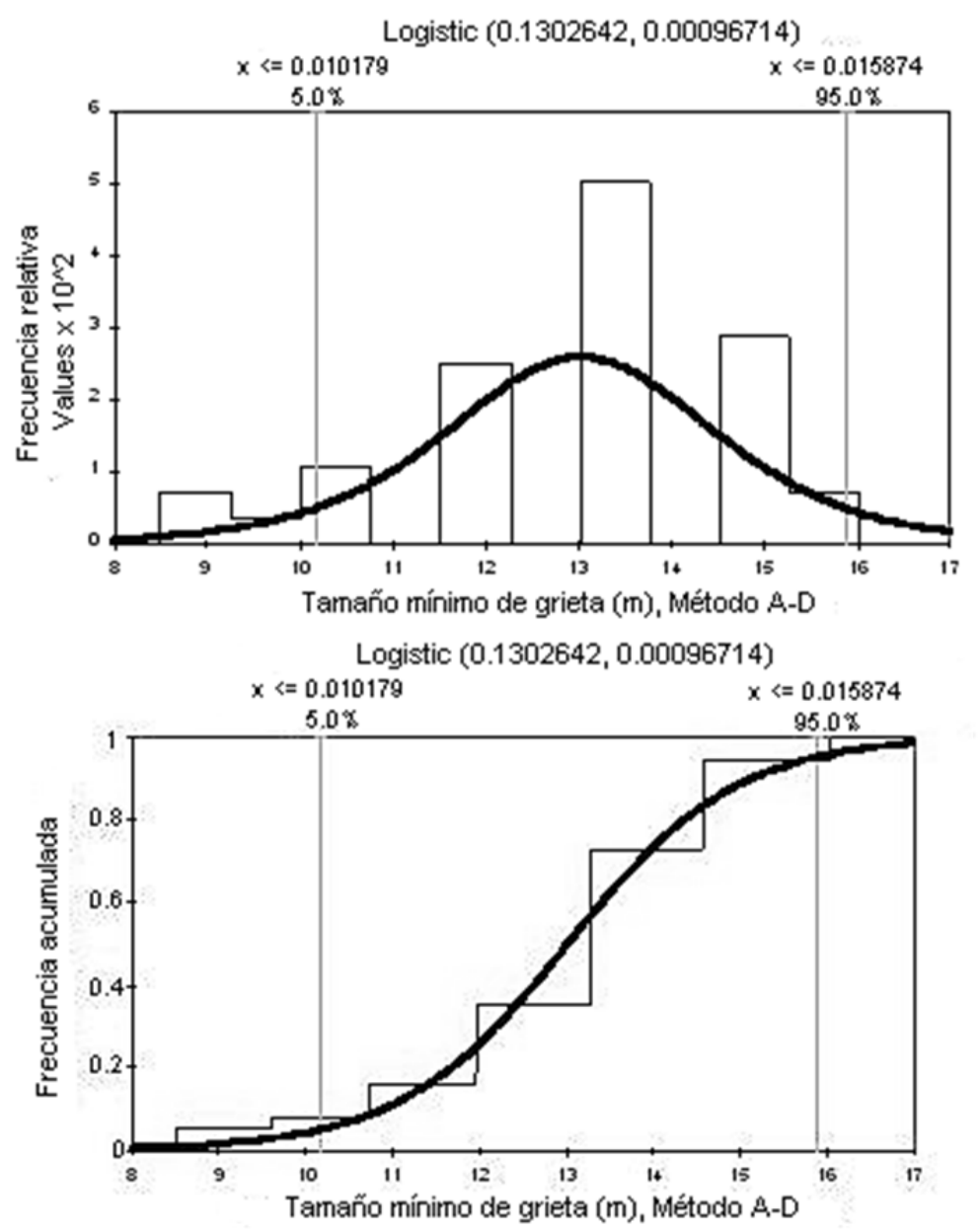




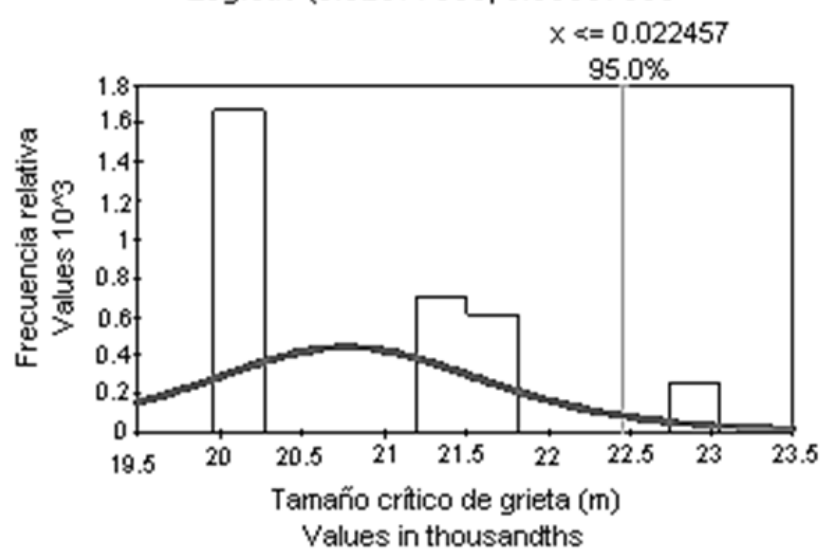

Logistic $(0.02077668,0.00057060$

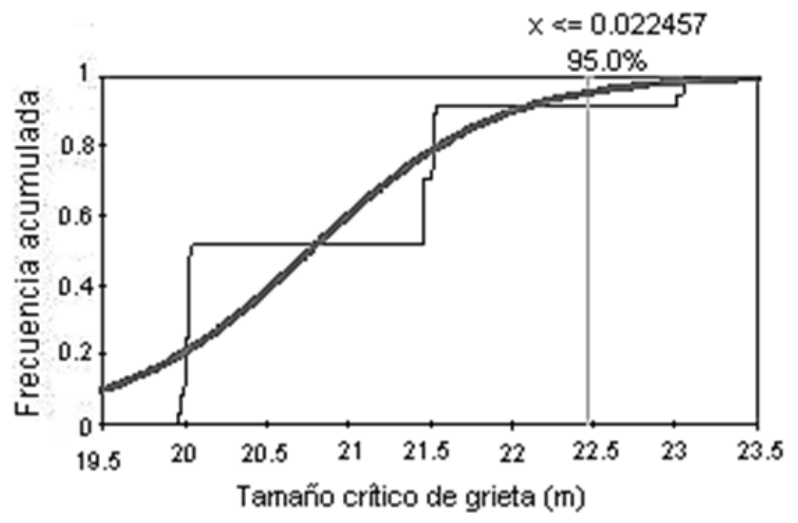

Figura N. 5 - Distribución logística que muestra el comportamiento de tamaños de grietas del Oleoducto

En general, las curvas que muestran el comportamiento de la resistencia residual tienden a disminuir su pendiente a mayor tamaño de grieta. Esto hace que si al detectar una grieta se opta por la disminución de la carga, por ejemplo, en 50\%, el incremento en la vida remanente es del mismo orden, mientras que si la curva de resistencia residual se eleva $25 \%$, la vida aumenta $100 \%$ (González, 2004). Además de esta diferencia, la reducción de la 
carga puede significar fuertes pérdidas por la disminución de la capacidad de producción. Por ende, los esfuerzos para la extensión de vida deben ir dirigidos al reforzamiento o uso de mejores materiales (Budinski, 1992).

En un análisis por separado para la iniciación y el crecimiento de la grieta se justifica por el diferente nivel de la tensión en el fondo de entalla antes y después de iniciarse la fisura, $\sigma_{f}$ y $\sigma_{f g}$ (Buckley, 2005), (Orowan, 1948). Se ha estudiado mucho la velocidad de propagación de la grieta encontrándose correlación con el incremento del factor multiplicador de tensiones, $\Delta K_{I}$ entre los estados extremos del esfuerzo aplicado al ducto (Ferrer \& Amigo, 2005) (Figura N. ${ }^{\circ}$ 6).

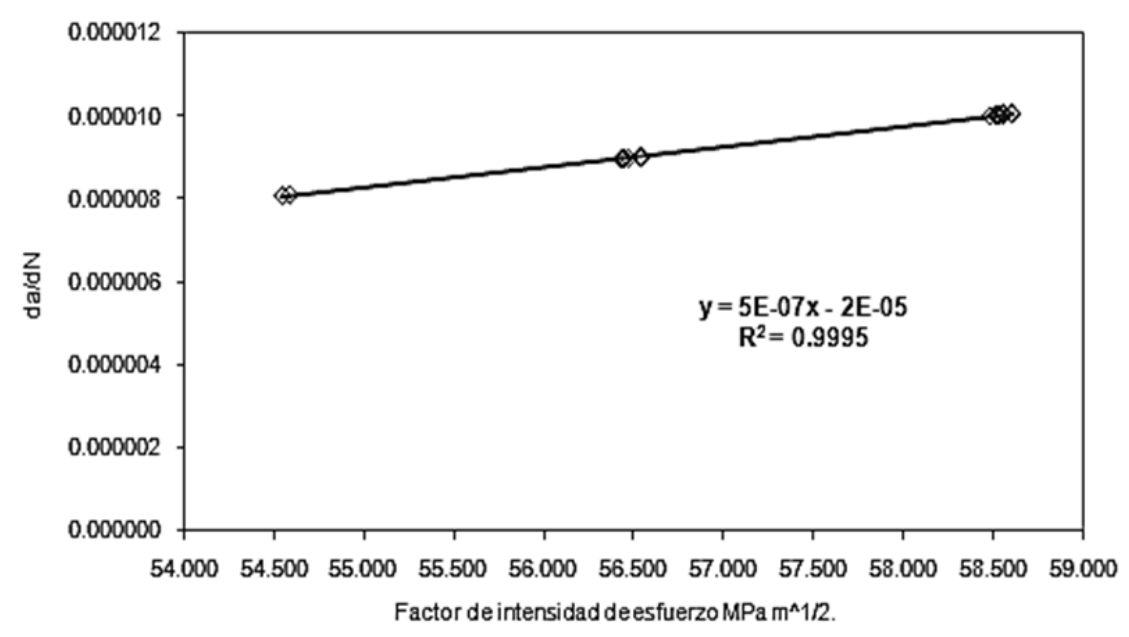

Figura N. ${ }^{\circ} 6$ - Tenacidad a la fractura vs vida del Oleoducto

La rapidez de propagación de las grietas presentes en el Oleoducto tiene un comportamiento descripto por la curva de distribución logística; la curva de densidad de probabilidad y de distribución acumulada correspondientes a dicha velocidad de propagación se muestra en la figura N. 7 . 


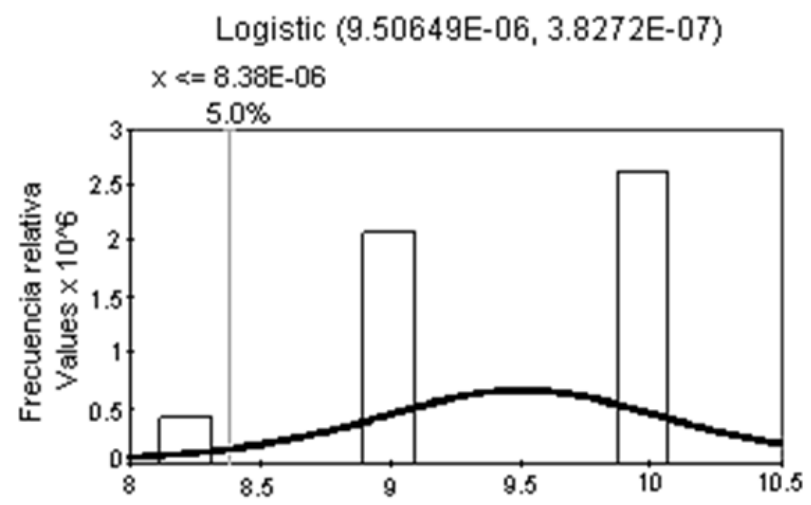

Rapidez de progación grietas (mm/ciclos), Método A-D

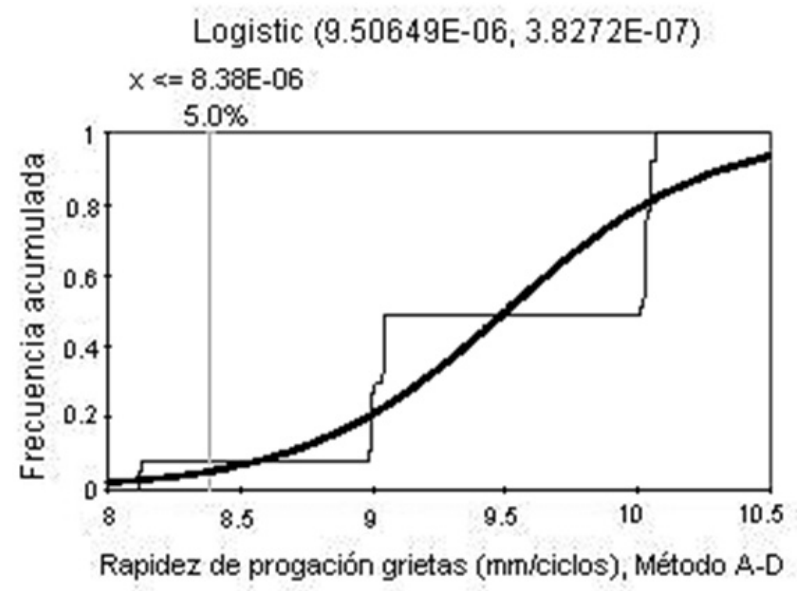

Figura N. 7 - La rapidez de propagación de grietas en el Oleoducto presenta una curva de distribución logística

Por otro lado, el modelo matemático de París $\left(d a / d N=C(\Delta K)^{n}\right)$, correlaciona el crecimiento linealizado de cada grieta del oleoducto, etapa II, con la pendiente $n$. La etapa II queda entre la I, como crecimiento estable rápido, y la III, de crecimiento inestable y rápido que se ha justificado en la teoría de fatiga. La integración de esta ecuación permite el cálculo del número de ciclos entre dos estados en el proceso de crecimiento de la grieta de fatiga (Ferrer \& Amigo, 2005). En la figura N. 6 queda implícito el concepto 
de límite de fatiga que corresponde a los valores de $\Delta K<\Delta K_{I S \text {. }}$ Como el cálculo de los ciclos de fatiga (vida) de la figuras 4 y 6 , hasta la fractura se realizó por integración de la ecuación de París entre los valores: En el caso de la vida final del ducto: (a) dimensión inicial de la grieta, $a_{0}$, y (b) la dimensión final $a_{c}$, equivalente a la tenacidad estática del material $K_{I C}$, y en el caso de la vida útil de la tubería, el cálculo se realizó con las dimensiones de la grieta mínima $a_{\min }$ y la grieta critica $a_{c}$, respectivamente.

\section{Conclusiones}

Debido a que el oleoducto está en servicio, está sometido a la acción de fuerzas y acciones agresivas provenientes tanto de las cargas de servicio como del ambiente, su estado físico se deteriora conduciendo a la disminución de la resistencia del material de que está hecho, pérdida de material de la sección transversal o del espesor y la aparición de defectos como socavaduras lo que puede reducir su la vida útil.

Cuando se detecta una grieta, la vida remanente de un oleoducto puede ser extendida de dos maneras: una es reduciendo la carga de servicio, lo cual hará que el tamaño crítico de grieta aumente, alargando la vida de dicho tubo; la otra es incrementando la resistencia del material o insertando un refuerzo, lo que eleva la curva de resistencia residual y hace que el tamaño crítico de grieta aumente. Para que se reduzca la vida útil, se requiere que el daño sea acumulativo, es decir, que aumente a través del tiempo. La rapidez de deterioro del ducto en servicio; por lo general, la acumulación de daño es inicialmente rápida por el hecho de ser una tubería grande y soterrada, por lo que las formas de daño tendrán uno o varios efectos adversos, siendo los más significativos la reducción de la vida útil y la reducción de la capacidad de carga de trabajo, ya que la disminución de la capacidad de carga de servicio sin señales evidentes o detectables es la causa, en muchos casos, de fallas o averías inesperadas. 
Por medio de este análisis de integridad se determinó la tensión máxima que puede resistir el ducto a un mínimo espesor donde la fluencia no afecta el estado de la estructura y la magnitud del daño tolerable para el cual se calculó la presión, las tensiones y el tamaño de las grietas iniciales y críticas, establecido a partir de estas la vida útil del oleoducto. Asimismo, se vio que los parámetros de resistencias de integridad del oleoducto muestran un comportamiento descripto por una distribución logística. Sin embargo, el deterioro superficial causado por las cargas de trabajo en dicho componente presenta un comportamiento regido por una función de distribución $\log$-logística.

\section{Referencias bibliográficas}

Anderson, T. L. (1995). Fracture Mechanics: Fundamentals and Applications. New York: CRC Press.

Askeland, D., \& Phulé, P. (2006). Science and Engineering of the Materials. (5 $5^{\text {ta. }}$ ed.). México: Tomson Brooks.

Balankin, A. S., \& O. Susarrey. (1999). A New Statistical Distribution Function for Self-Affine Crack Roughness Parameters. Philosophical Magazine Letters, 79(6).

Balankin, A. S.; Hernández, L.; Urriolagoitia, G.; Susarrey, O.; Gonzáles, J. M.; Martínez, J. (1999). Probabilistic Mechanics of Self-Affine Cracks. Proceedings of the Royal Society, 455 (7).

Bell, C., \& Johnson, D. (2007). Resistencias de materiales. (4ta. ed.). México: McGraw-Hill.

Buckley, C. P. (2005). Material Failure. Lecture Notes. Oxford: University of Oxford.

Budinski, K. (1992). Engineering Materials Properties and Selection. (5ta. ed.). Washington: Prentice-Hall. 
Budynas, R., \& Nisbett, J. K. (2008). Diseño en ingeniería mecánica de Shigley. (8ta. ed.). México: McGraw-Hill.

Erdogan, E. (2000). Fracture Mechanics. International Journal of Solids and Structures, 27, 171-183.

Ferrer Jiménez, C., \& Amigo Borrás, V. (2005). Tecnología de materiales. México: Alfa y Omega.

González Vázquez, J. L. (2008). Propagación de grietas por fatiga en soldaduras SAW de Tubo de Acero API 5L en Probetas SEN Curvas no Estándar en la Dirección Corta Radial. (Tesis de maestría), ESIQUIE-IPN, México, D. F.).

González, J. L. (2004). Mecánica de la fractura. México: Limusa.

Irwin G. (1957). Analysis of stresses and strains near the end of a crack traversing a plate. Journal of Applied Mechanics 24, 361-364.

Mangonon, P. L. (2001). Selección y diseño de ciencia de los materiales. México: Pearson Educación.

Martínez Lorenzo, Susarrey Orlando, L. C.; Balankin; Martínez José, A. (2000). Mecánica de la fractura probabilística para análisis de oleogasducto. Revista Científica (en línea): http://www.mfractal.esimez.ipn.mx/integrantes/balankin/b alankin_publications/42_difusion/42_11.pdf.

Mendenhall, W., \& Sincich, T. (1999). Probability and Statistics for Engineering and Science. (4ta. ed.). Mexico: PrenticeHall.

Orowan, E. (1948). Fracture and strength of solids. Reports on Progress in Physics XII, 185-232.

Palisade Corporation. (2002). @Risk Risk Analysis and Simulation. [CD-ROM. 4.5]. New York: Palisade Corporation. 
Shigley, J. E., \& Mischke, C. (1990). Diseño en ingeniería mecánica. (4ta. ed.) México: McGraw-Hill.

Smith, W. F., \& Hashemi, J. Basis of the Science and Engineering of Materials. (4ta. ed.). México: McGraw-Hill.

Walpole, R., \& Myers, R. (1992). Probabilidad y estadística. (4ta. Ed.). México: McGraw-Hill.

\section{Reconocimientos}

Se le agradece a la empresa FALCONDO por su gran aporte en el trabajo experimental y el apoyo brindado por el Grupo de Mecánica Fractal de la ESIMEZ del Instituto Politécnico Nacional de México D. F. 


\section{José Luis Soto Trinidad}

Es profesor, pleno investigador en el Instituto Tecnológico de Santo Domingo (INTEC) en ingeniería mecánica y realiza investigaciones en las líneas de diseño mecánico, mecánica de la fractura, estudio de materiales y fractales conjuntamente con investigadores del Instituto Politécnico Nacional de México. También imparte docencia como profesor de ingeniería en la UASD. Tiene varias publicaciones en revistas indexadas y ha participado como ponente en varios congresos internacionales sobre ingeniería. En junio de 2000, se graduó de Ingeniero Electromecánico Mención Mecánica por la Universidad Autónoma de Santo Domingo (UASD). En marzo de 2004, se graduó de maestro en Ciencias en Ingeniería Mecánica-Diseño Mecánico por la Escuela Superior de Ingeniería Mecánica y Eléctrica del Instituto Politécnico Nacional de México DF. En Julio de 2007, obtiene su doctorado en Ciencias en Ingeniería Mecánica en esta institución.

Email: sototjoseluis@yahoo.es

Recibido: 15/02/2013

Aprobado: 02/05/2013 